\title{
Perinatal and psychosocial circumstances associated with risk of attempted suicide, non- suicidal self-injury and psychiatric service use. A longitudinal study of young people
}

\author{
Robert Young ${ }^{1 *}$, Vincent Riordan ${ }^{2}$ and Cameron Stark ${ }^{3}$
}

\begin{abstract}
Background: Past studies using large population based datasets link certain perinatal circumstances (birth weight, parity, etc) with mental health outcomes such as suicide, self-harm and psychiatric problems. Problematically, population datasets omit a number of social confounds. The aim of this study is to replicate past research linking perinatal circumstances and mental health (suicidality and use of psychiatric services) and to determine if such associations remain after adjusting for social circumstances.
\end{abstract}

Methods: A longitudinal school-based survey of 2157 young people (surveyed at age 11, 13, 15) followed up in early adulthood (age 19). At age 11 parents of participants provided information about perinatal circumstances (birth weight, birth complications, etc.) and psychiatric service use. Participants provided data about their mental health at age 15 (attempted suicide, suicidal thoughts) and at ages 19 (self-harm, psychiatric service use). In addition, data were collected about their social and psychosocial circumstances (gender, deprivation, religion, sexual behaviour, etc.).

Results: Predictably, social factors were linked to mental health outcomes. For example, those with same sex partners were more likely (OR 4.84) to self-harm than those without a same sex partner. With a single exception, in both unadjusted and adjusted models, perinatal circumstances were not or only marginally associated with mental health outcomes. The exception was the number of birth complications; young people with two or more complications were approximately 2-3 times more likely than those without complications to use psychiatric services.

Conclusions: While we failed to replicate results found using large population based datasets, some of our results are compatible with prior research findings. Further, evidence from this study supports the influence of perinatal circumstances (birth complications) on later psychiatric problems, or at least higher than expected contact with psychiatric service.

\section{Background}

Mental health in adulthood may be significantly influenced by earlier life environments $[1,2]$. The importance of the childhood and adolescent environment has long been recognised. Attachment theory [3] emphasises the importance of the earliest relationships, and robust

\footnotetext{
* Correspondence: robert@sphsu.mrc.ac.uk

'MRC Social and Public Health Sciences Unit, University of Glasgow, 4

Lilybank Gardens, Glasgow G12 8RZ, Scotland, UK

Full list of author information is available at the end of the article
}

associations have been demonstrated between mental ill-health in adulthood and such childhood adversities as parental separation [4] and childhood maltreatment or abuse [5-9]. In more recent years variables from the perinatal environment have also been demonstrated as associates of adult mental health outcomes. Adverse outcomes have been linked with factors such as antenatal maternal malnutrition [10], antenatal maternal stress [11], infant malnutrition [12], low birth weight, younger maternal age and increasing birth order [13-16]. These 
observations, in particular those involving the antenatal environment, suggest a possible biological basis, possibly involving epigenetic developmental processes [17].

Most mental ill-health has a complex multifactorial aetiology and any environmental associations may be of clinical or public health importance in that they may indicate possible areas for preventative intervention. However, because of the possibility of unidentified confounding variables, it is important to attempt to distinguish between factors which, although linked to increased risk, do not in themselves mediate that risk (risk indicators), as opposed to factors which directly contribute to the causal pathway leading to the development of mental illness (risk mediators). For example, a number of studies have suggested that much of the association between parental separation and later adverse mental health outcomes can be accounted for by other psychosocial variables, such as socioeconomic deprivation, exposure to parental conflict and poor child-parent relationships [18-20].

The relative importance of very early influences, such as those occurring in the perinatal period, compared with later variables, such as adolescent or early adult circumstances is unclear [21]. Much of the existing evidence regarding perinatal variables relies on large population based datasets. Whereas these have the advantage of avoiding recall bias and yielding high statistical power, they may exaggerate the significance of variables such as maternal demographics, on which data are readily available, whilst failing to control for plausible confounders, such as breastfeeding or family structure, on which data (from large datasets) tends to be poor or absent.

\section{Aims}

The aim of the study is to examine, using self reported data, the association between perinatal and psychosocial risk factors and mental health outcomes, thereby testing the hypothesis that both perinatal circumstances and more chronologically proximate psycho-social circumstances are risk mediators, (rather than indicators) of mental ill-health in early adulthood. The study allowed a number of variables from different stages of development to be brought together in a single multivariable analysis whilst also replicating previous studies using an alternative study design.

\section{Methods}

The material for the study is drawn from a Scottish longitudinal community health and lifestyle survey of young people, administered first in-school via questionnaire (ages 11, 13, and 15), with an additional parental questionnaire at age 11, and then in the post-school period by nurse interview at age 19 . The focus here is on mental health outcomes at age 15 (1999) and age 19 (2003) within the framework of the 'West of Scotland 11 to 16 Study/16+' [22]. The study received approval from Glasgow University's Ethics Committee, participating Education Authorities and schools, and informed consent was obtained from the parents of all participants via 'opt-out' consent forms at ages 11, 13 and 15, verbal consent from participants at each wave and written consent at age 19.

Due to the school-based nature of the sample the sampling scheme involved several elements to ensure a representative sample at both the primary and secondary school stages [23]. Briefly, the survey used a reverse sampling procedure which randomly selected 43 secondary schools stratified by religious denomination and deprivation, with a separate stratum for independent vs. local authority run schools. These 43 secondary schools were used to select a random sample of 135 primary schools, comprising 'feeder schools', together with those making a high number of placing requests. From these primary schools, classes were randomly selected with all pupils in the classes eligible to participate. Of the 2793 pupils who attended the 43 targeted secondary schools, 2586 (93\%) participated in the baseline (age 11) survey. At age 13, the number of participants reduced to 2371 (85\%), and by 15 to 2196 (79\%). At age $15,1,860(67 \%)$ of respondents completed a psychiatric interview [24], which included questions about suicidal thoughts and attempts. As expected losses in the postschool period substantially reducing the sample size at age 19 to 1256 (45\%). Full details of the sampling strategy are available elsewhere [23].

At age 11 the sample was representative (in terms of sex and social class composition) of 11 year olds in the study area [25]. Differential attrition made later waves less representative, with attrition greater among lower social class groups, school truants, pupils of lower ability and with greater emotional and behavioural problems. To compensate for these biases, a weighting scheme was derived [25]. Use of these weights did not substantively alter any of the results presented here. The data used in this paper refer to 2196 pupils in their final year of compulsory education in 43 mainstream secondary schools in the Glasgow area, 1256 of whom provided information when aged 19. Parents provided information on perinatal circumstances such as maternal age and birth weight and on pupils' religious background and family socioeconomic status via a questionnaire in the first wave (age 11) of the study. The final sample included 16 twin births, but excluded adopted children ( $\mathrm{n}=39$ ), reducing the sample to 2157 .

\section{Measures}

In 1996 (pupils aged 11) parents were asked questions about the child's birth history and perinatal 
circumstances. This included questions on maternal parity, categorised as first or later born; family size (including parents), categorised as 2 or more family members; subjective recall of the size of the baby (small, average, or large); birth weight (in grams), categorized $<2500$, 2500-3249, 3250-3749, or 3750g+; number of birth complications (e.g. breech birth, pre-eclampsia, etc), categorized none, one, two or more; birth spacing (both prior to and post index birth), categorised as under 2 years, 25 years, or $6+$ years between births (or singleton); maternal age, categorised age 15-19, 20-24, 25-29, 3034 , or 35-46. Finally, parents provided information on breastfeeding practices, categorised as none, breastfeeding for less than six months, or six months or longer. Whenever feasible we derived categories compatible with established cut-points or expert recommendations. For example, in relation to the six month cut-point for breastfeeding, the American Academy of Pediatrics recommendation is 'exclusive breastfeeding is sufficient to support optimal growth and development for approximately the first 6 months of life' [26].

Several background factors relevant to either sociodemographic circumstances or psychiatric outcomes at age 11 and age 15 were recorded. At age 11 an area deprivation score, range 1 (least) to 7 (most deprived), was derived from pupils' postal codes using the 'Carstairs' [27] index, a standard measure based upon census data. Social class of the head of household was derived from parental questionnaires completed at wave one (age 11), coded using the standard UK classification system [28] and categorized as non-manual or manual. Religious affiliation was obtained from parents and categorized, Church of Scotland (Protestant), Catholic, Muslim, other (Jewish, Methodist, Baptist, etc) and 'none, atheist/agnostic'. At age 15, pupil's family structure was coded as 2-parent, 1-parent, reconstituted (one 'birth' parent and new partner) or other (relative, foster parent, or other carer). Principal component (varimax) analysis of the (age 15) 8-item Brief Parental Bonding Instrument [29], produced two scales representing perceived (low) parental care, e.g. 'My parents help me as much as I need' (reversed) and (high) control, e.g. 'My parents treat me like a baby'.

Sexual orientation is an established psychosocial risk factor for suicide and other psychiatric disorders and early gender nonconformity is a strong predictor of future sexual orientation [30,31]. Gender diagnosticity (GD) is an established measure of gender nonconformity, previously measured in this cohort at age 15 and linked with poor psychological wellbeing [30]. GD score is the probability of belonging to a particular gender, as predicted by the logistic regression of multiple items of gendered behaviours, interests and hobbies. The result is a score (0-1 scale) of how 'typically' male or female an individual is (within a limited domain) compared to their peers. An arbitrary cut-point of 0.1 (10\%) has been used to indicate 'gender conformist' (top 10\%), 'Gender typical' (middle 80\%) and gender nonconformists (bottom 10\%) respectively. At age 19, participants were asked 'Have you ever had any kind of sexual experience or sexual contact with someone of the same gender as yourself' and asked to response 'yes' or 'no' using a 'show card procedure' designed to reduced response bias. While not a comprehensive measure of sexual preference, this item is arguably a good indicator of samesexual orientation and behaviour.

Several dichotomous mental health outcomes were reported at ages 15 and 19 . At age 15 participants were asked if they had ever seriously thought about taking their own life and if they had attempted to do so, categorised as suicidal thoughts and attempted suicide respectively, with a positive response to either question classified as 'suicide risk'. At age 19 participants were asked if they had ever 'tried to hurt or harm yourself deliberately' and to indicate which method(s) the had used, such as cutting, scratch or scoring or burning; a positive response was classified as Nonsuicidal Self-Injury NSSI. To assess previous psychiatric problems, parents were asked about the child's use of psychiatric services until age 11 and at age 19 participants were asked about their use of psychiatric services since age 11. Due to both budget and ethical constrains suicidality was only measured at age 15 and 19', self-harm at age 19 and psychiatric contact only measured at age 11 and 19.

\section{Statistical analysis}

The analysis used logistic regression to determine the association between perinatal and psychosocial circumstances and age 15 and age 19 psychiatric outcomes. Analysis was conducted both unadjusted and mutually adjusted for covariates. We constructed weights to compensate for differential attrition (21), but use of these weights did not alter results. The influence of missing data was further explored by comparing results for models using complete data only and multiple imputation methods. Multiple imputation was implemented using the STATA 'ice' procedure and included all variables from the relevant model, with separate imputation runs for every analysis. Birth size was included as a supplementary variable, because it contained information useful in estimating birth weight. Categorical variables were imputed using logit or multiple logit, continuous variables using regression and deprivation using ordinal logit commands. Ten imputed datasets were used to calculate the final combined estimates. Although the results for each method were not substantively different, we report results based on multiple imputation. Results from all other models are available upon request. 


\section{Results}

Univariate results

Table 1 reports descriptive statistics and the number of cases with missing data for the perinatal covariates; table 2 reports these for psychosocial covariates and psychiatric outcomes. Given the age and nature of the

\section{Table 1 Descriptive statistics for perinatal covariates}

\begin{tabular}{|c|c|c|}
\hline Perinatal covariates ${ }^{\text {all @11p }}$ & $\mathbf{N}$ & (\%) \\
\hline \multicolumn{3}{|l|}{ Maternal parity } \\
\hline 0 first born & 989 & 45.9 \\
\hline *1 second born & 784 & 36.3 \\
\hline${ }^{*} 2+$ third or later & 384 & 17.8 \\
\hline \multicolumn{3}{|c|}{ Family size (total at age 11 ) + Twins } \\
\hline 2 & 247 & 11.5 \\
\hline *3 & 1001 & 46.4 \\
\hline${ }^{*} 4+$ & 909 & 42.1 \\
\hline \multicolumn{3}{|l|}{ Birth size $[M V=20]$} \\
\hline Small & 293 & 13.7 \\
\hline Average & 1458 & 68.2 \\
\hline Large & 386 & 18.1 \\
\hline \multicolumn{3}{|l|}{ Birth weight $(g)[M V=96]$} \\
\hline$<2500$ & 133 & 6.5 \\
\hline $2500-3249$ & 719 & 34.9 \\
\hline $3250-3749$ & 801 & 38.9 \\
\hline $3750+$ & 408 & 19.8 \\
\hline \multicolumn{3}{|l|}{ Birth complications [MV = 15] } \\
\hline 0 & 1499 & 70.0 \\
\hline 1 & 360 & 16.8 \\
\hline${ }^{*} 2$ & 189 & 8.8 \\
\hline *3 or more & 94 & 4.4 \\
\hline \multicolumn{3}{|l|}{ Birth spacing - Prior birth } \\
\hline Under 2 years & 346 & 16.0 \\
\hline $2-5$ years & 502 & 23.3 \\
\hline $6+$ years or singleton & 1309 & 60.7 \\
\hline \multicolumn{3}{|l|}{ Birth spacing - Next birth } \\
\hline Under 2 years & 429 & 19.9 \\
\hline $2-5$ years & 510 & 23.6 \\
\hline $6+$ years or singleton & 1218 & 56.5 \\
\hline \multicolumn{3}{|c|}{ Maternal age (years) $[\mathrm{MV}=79]$} \\
\hline 15-19 & 139 & 6.7 \\
\hline $20-24$ & 626 & 30.1 \\
\hline $25-29$ & 764 & 36.8 \\
\hline $30-34$ & 421 & 20.3 \\
\hline $35-46$ & 128 & 6.2 \\
\hline \multicolumn{3}{|l|}{ Breastfeeding [MV = 12] } \\
\hline None & 1333 & 62.1 \\
\hline Less than 6 months & 463 & 21.6 \\
\hline 6 months or more & 349 & 16 \\
\hline
\end{tabular}

Baseline $N=2157$, final sample excludes adopted children i.e. only natural mother.

MV = missing values; * Adjacent category collapsed for analysis; +Twins = includes 16 twin births.

$@ 11 p=$ parental figure provided information when participants age 11
Table 2 Descriptive statistics for psychosocial covariates and psychiatric outcomes.

\begin{tabular}{|c|c|c|}
\hline Social and demographic covariates & $\mathbf{N}$ & (\%) \\
\hline \multicolumn{3}{|l|}{ Sex ${ }^{@ 11}$} \\
\hline Female & 1076 & 49.9 \\
\hline Male & 1081 & 50 \\
\hline \multicolumn{3}{|l|}{ Social Class ${ }^{@ 11 p}[\mathrm{MV}=376]$} \\
\hline Manual & 860 & \\
\hline Non-manual & 921 & 51 \\
\hline \multicolumn{3}{|l|}{ Family structure age $15^{@ 15}$ [MV = 288] } \\
\hline 2-parent & 1410 & \\
\hline 1-parent, & 274 & 14.7 \\
\hline Reconstituted/Other & 185 & 9.9 \\
\hline \multicolumn{3}{|c|}{ Same sex partner by $19{ }^{\circledR 19}[\mathrm{MV}=1066]$} \\
\hline No & 1045 & \\
\hline Yes & 46 & \\
\hline
\end{tabular}

Gender nonconforming behaviour at age 15

(Gender Diagnosticity) ${ }^{@ 15}$ [MV = 289]

Gender conformist (10\%) $179 \quad 9.6$

Gender typical (80\%) $1500 \quad 80.3$

Gender nonconformist (10\%) $189 \quad 10.1$

Religion ${ }^{\circledR 11 \mathrm{p}}$ (MV = 19)

$\begin{array}{lll}\text { Protestant } & 913 & 42.7\end{array}$

$\begin{array}{lll}\text { Roman Catholic } & 721 & 33.7\end{array}$

*Muslim/slam $\quad 57 \quad 2.7$

*Other (Baptist, Jewish, etc) $\quad 125 \quad 5.8$

$\begin{array}{lll}\text { None/Atheist/Agnostic } & 322 & 15.1\end{array}$

\begin{tabular}{lll}
\hline Psychiatric outcomes & N & (\%)
\end{tabular}

Suicidal thoughts past year at age $15{ }^{\circledR 15}[\mathrm{MV}=522]$

No thoughts $\quad 1513 \quad 94.3$

$\begin{array}{lll}\text { Thoughts } & 92 & 5.7\end{array}$

Attempted suicide by age $15{ }^{@ 15}[\mathrm{MV}=522]$

$\begin{array}{lll}\text { No attempt } & 1511 & 94.1\end{array}$

Attempt $\quad 94 \quad 5.9$

Risk: Any ideation/attempt by age $15{ }^{@ 15}[\mathrm{MV}=522]$

None $\quad 1460 \quad 91.0$

Attempt or ideation $\quad 145 \quad 90$

NSSI by age $19{ }^{@ 19}[\mathrm{MV}=1066]$

No NSSI $1013 \quad 92.9$

NSSI $\quad 78 \quad 7.1$

Psychiatric services by age $11{ }^{@ 11 \mathrm{p}}[\mathrm{MV}=11]$

$\begin{array}{lcc}\text { Not used } & 2064 & 96.2 \\ \text { Used } & 82 & 3.8\end{array}$

Psychiatric services since age $11{ }^{\circledR 19}[\mathrm{MV}=1066]$

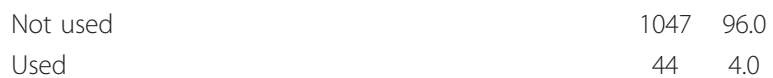

Psychiatric services ANY ${ }^{11 p}$, @19 $[\mathrm{MV}=1070]$

Not used

$1017 \quad 93.6$

\begin{tabular}{lcc} 
Used & 70 & 6.4 \\
\hline Continuous covariates & M & SD \\
\hline Area deprivation (depcat) ${ }^{\circledR 11}[\mathrm{MV}=\mathbf{2 9 9}]$ & 4.11 & 1.90 \\
(Lack of) Parental Care ${ }^{\circledR 15}[\mathrm{MV}=\mathbf{2 8 8}]$ & 5.59 & 1.59 \\
Parental Control ${ }^{\circledR 15}[\mathrm{MV}=\mathbf{2 8 8}]$ & 7.85 & 1.09 \\
\hline
\end{tabular}


Baseline $N=2157$, final sample excludes adopted children i.e. only natural mother.

MV = missing values; $*$ Adjacent category collapsed for analysis

$@ 11$ = participant provided information at age 11; ${ }^{@ 15}=$ information provided at age $15 ;{ }^{@ 19}=$ information provided at age $19 ;{ }^{@ 11 p}=$ parental figure provided information when participants age 11

cohort, the observed pattern of results is typical for this type of survey data.

\section{Suicidality and self-harm}

Table 3 shows the unadjusted associations between covariates and rate of attempted suicide, suicide risk (either ideation or attempt) at age 15 and NSSI by age 19. As expected, several psychosocial covariates are associated with significant increases in the odds in attempted suicide, suicide risk or NSSI. For example, compared to males, females show approximately a threefold increase in the odds of attempting suicide or having suicidal thoughts, although adjusting for covariates slightly attenuates this. Being part of a reconstituted family was also a risk factor for all suicidality and NSSI outcomes and lack of parental care was another. Compared to the 'gender typical' group, 'gender nonconformists' had a twofold increase in the odds of attempted suicide or NSSI, although this was non-significant in the adjusted model. In the unadjusted model, young people who had at least one same sex partner show nearly a fivefold increase in odds of NSSI, compared to those who never had a same sex partner; this association remained strong in the adjusted model $(\mathrm{OR}=3.85)$. Perinatal variables were unrelated or only marginally associated with suicidality or self-harm.

\section{Use of psychiatric services}

Table 4 shows the unadjusted associations between covariates and use of psychiatric services before age 11 and between age 11 and age 19. We found the expected associations between psychosocial covariates and use of psychiatric services. For example, compared to males, females show significantly lower odds (OR 0.38) of using services before age 11, but significantly higher odds (OR 2.07) of service use after age 11; including covariates made little difference to these results. Young people from reconstituted families and those with lack of parental care had a higher likelihood of using services, although his was not always statistically significant. With the exception of birth complications, most perinatal variables were again unrelated, or only marginally associated, with use of psychiatric services. Compared to those without birth complications, those with two or more birth complication have substantially increased odds (OR 2.3-3.8) of psychiatric contact, either before or after age 11, although in the adjusted model this was attenuated (OR 1.7-2.9), but still significant for service contact both before age 11 and for lifetime psychiatric service contact.

\section{Discussion}

This study demonstrated a significant and sizable association between the number of birth complications and psychiatric service contact. If replicated this is important, because within the current perinatal circumstances literature birth complications is a relatively new focus of study of substantial effect size, compared to other perinatal factors. As expected we demonstrated a significant association between adverse mental health outcomes and adolescent psychosocial variables such as reconstituted family structure, perceived lack of parental care and having a same sex partner. Previous observations regarding perinatal variables were not replicated, but given the currently limited evidence-base, consisting of a handful of large population based studies, there are a number of potential explanations for the general lack of association with mental health outcomes.

\section{Associations between birth complications and psychiatric service use}

A significant association was observed between birth complications and contact with psychiatric services before the age of 11 . This is consistent with previous work linking birth trauma and developmental disorders [32], although the potential influence of parental behaviour should be borne in mind and this is discussed in detail later. Although still somewhat controversial, several authors link the concepts of general neurological, potentially birth-related damage, or perinatal stress to the development of psychiatric or mental health problems using a variety of terms such as Minimal Brain Dysfunction (see review by Rutter [33]) and more recently, deficits in attention, motor control, perception (DAMP). In relation to DAMP the attention has focused on learning and language difficulties, autistic spectrum and attention deficit/hyperactivity (ADHD) disorders, although more general psychopathology such as anxiety and depression have also been linked [34].

No relationship was observed between birth complications and suicidality in mid teens, although a statistically non-significant association was observed between such complications and NSSI at the age of 19, an association which increased following adjustment for background factors, suggesting that this perinatal variable may be a risk mediator. Such (weak) associations are consistent with well established links between obstetric complications and increased risk of adult psychosis [35], although links with affective disorders [36] or suicidality [37] have not been clearly established. We find a general association between obstetric complications and a crude measure of psychiatric problems (service contact), but any 
Table 3 Associations (odds ratios) between social, demographic, perinatal factors and suicide risk or self-harm.

\begin{tabular}{|c|c|c|c|c|c|c|}
\hline \multirow[b]{2}{*}{ Covariates } & \multicolumn{2}{|c|}{ Suicide attempt by age 15} & \multicolumn{2}{|c|}{$\begin{array}{l}\text { Suicide risk: } \\
\text { Suicidal ideation/attempt by age } 15\end{array}$} & \multicolumn{2}{|c|}{ NSSI by 19} \\
\hline & Unadjust & Adjusted & Unadjust & Adjusted & Unadjust & Adjusted \\
\hline \multicolumn{7}{|l|}{ Psychosocial } \\
\hline \multicolumn{7}{|l|}{ Sex @11 } \\
\hline Male & 1.00 & 1.00 & 1.00 & 1.00 & 1.00 & 1.00 \\
\hline Female & $3.20(1.93-5.30)$ & $3.06(1.79-5.22)$ & $2.77(1.91-4.03)$ & $2.69(1.83-3.96)$ & $1.32(0.89-1.97)$ & $1.25(0.82-1.89)$ \\
\hline \multicolumn{7}{|l|}{ Social class @11p } \\
\hline Non-manual & 1.00 & 1.00 & 1.00 & 1.00 & 1.00 & 1.00 \\
\hline Manual & $1.39(0.86-2.25)$ & $1.20(0.71-2.03)$ & $1.20(0.85-1.69)$ & $1.03(0.66-1.59)$ & $0.90(0.55-1.45)$ & $0.85(0.49-1.49)$ \\
\hline \multicolumn{7}{|l|}{ Family structure @15 } \\
\hline 2-parent & 1.00 & 1.00 & 1.00 & 1.00 & & 1.00 \\
\hline 1-parent, & $1.48(0.80-2.73)$ & $1.25(0.64-2.44)$ & $1.34(0.80-2.26)$ & $1.20(0.67-2.15)$ & $1.43(0.77-2.65)$ & $1.34(0.70-2.56)$ \\
\hline reconstituted/Other & $3.21(1.93-5.34)$ & $2.37(1.30-4.34)$ & $2.51(1.61-3.92)$ & $2.10(1.27-3.48)$ & $2.84(1.43-5.65)$ & $2.47(1.20-5.10)$ \\
\hline \multicolumn{7}{|l|}{ Same sex part ${ }^{@ 19}$} \\
\hline No & 1.00 & 1.00 & 1.00 & 1.00 & 1.00 & 1.00 \\
\hline Yes & $2.19(0.68-7.07)$ & $1.39(0.33-5.78)$ & $1.48(0.59-3.72)$ & $1.03(0.33-3.26)$ & $4.84(2.24-10.46)$ & $3.85(1.67-8.87)$ \\
\hline \multicolumn{7}{|l|}{ Gender conform @15 } \\
\hline Gender typical & 1.00 & 1.00 & 1.00 & 1.00 & 1.00 & 1.00 \\
\hline Gender conformist & $0.79(0.34-1.81)$ & $0.82(0.36-1.89)$ & $0.82(0.42-1.62)$ & $0.87(0.44-1.72)$ & $0.95(0.51-1.78)$ & $1.01(0.51-1.99)$ \\
\hline Gender nonconformist & $1.89(1.06-3.35)$ & $1.65(0.80-3.41)$ & $1.24(0.72-2.16)$ & $1.12(0.58-2.19)$ & $1.84(0.91-3.71)$ & $1.38(0.59-3.22)$ \\
\hline \multicolumn{7}{|l|}{ Religion @11p } \\
\hline Protestant & 1.00 & 1.00 & 1.00 & 1.00 & 1.00 & 1.00 \\
\hline Roman Catholic & $0.92(0.55-1.52)$ & $0.89(0.52-1.54)$ & $0.88(0.58-1.32)$ & $0.88(0.57-1.38)$ & $0.77(0.48-1.22)$ & $0.81(0.50-1.34)$ \\
\hline Other (Baptist, etc) & $0.99(0.49-1.99)$ & $1.15(0.54-2.44)$ & $0.66(0.34-1.28)$ & $0.72(0.36-1.47)$ & $1.17(0.64-2.11)$ & $1.28(0.63-2.60)$ \\
\hline None/Atheist/Agnostic & $1.09(0.57-2.08)$ & $0.97(0.49-1.91)$ & $0.91(0.54-1.56)$ & $0.84(0.48-1.46)$ & $1.11(0.54-2.29)$ & $1.01(0.46-2.21)$ \\
\hline Area deprivation @11 & $1.09(0.96-1.23)$ & $1.04(0.89-1.20)$ & $1.04(0.95-1.14)$ & $0.99(0.88-1.12)$ & $1.01(0.90-1.13)$ & $1.02(0.88-1.17)$ \\
\hline Lack of par Care ${ }^{@ 15}$ & $1.33(1.19-1.49)$ & $1.30(1.15-1.47)$ & $1.34(1.21-1.49)$ & $1.33(1.18-1.49)$ & $1.32(1.18-1.48)$ & $1.30(1.16-1.46)$ \\
\hline Parental control @15 & $1.15(0.96-1.37)$ & $1.18(0.98-1.43)$ & $1.10(0.94-1.29)$ & $1.13(0.96-1.33)$ & $0.99(0.82-1.19)$ & $0.97(0.79-1.18)$ \\
\hline
\end{tabular}

Maternal parity

0

1 or more

Family size

2

3 or more

Birth weight (g)
$<2500$
2500-3249
3250-3749
$3750+$

$\mathrm{N}$ birth complications

0
1
2 or more

Birth spacing (prior)

Under 2 years

2-5 years

$6+$ years or singleton

Birth spacing (later)

Under 2 years

2-5 years
1.00

$1.11(0.70-1.76)$

1.00

$0.78(0.45-1.34)$

$1.44(0.63-3.30)$

$1.12(0.71-1.77)$

1.00

$0.74(0.42-1.30)$

1.00

$0.88(0.49-1.58)$

$0.90(0.47-1.74)$

1.00

$0.82(0.45-1.49)$

$1.02(0.60-1.72)$

1.00

$0.73(0.39-1.38)$
1.00

$1.67(0.60-4.65)$

1.00

$1.03(0.47-2.29)$

$1.46(0.56-3.84)$

$1.13(0.70-1.84)$

1.00

$1.04(0.58-1.88)$

1.00

$0.97(0.53-1.78)$

$1.00(0.49-2.02)$

1.00

$0.88(0.46-1.68)$

$0.91(0.48-1.74)$

1.00

$0.81(0.42-1.57)$
1.00

$1.06(0.70-1.60)$

1.00

$0.96(0.58-1.58)$

$1.30(0.65-2.58)$

$1.08(0.75-1.55)$

1.00

$0.81(0.51-1.29)$

1.00

$0.71(0.43-1.16)$

$0.84(0.50-1.40)$

1.00

$1.00(0.59-1.67)$

$1.03(0.64-1.65)$

1.00

$0.80(0.46-1.40)$
1.00

$1.44(0.65-3.20)$

1.00

$1.19(0.61-2.30)$

$1.39(0.66-2.94)$

$1.09(0.74-1.61)$

1.00

$1.03(0.63-1.70)$

1.00

$0.74(0.44-1.25)$

$0.90(0.51-1.58)$

1.00

$1.06(0.61-1.84)$

$1.00(0.58-1.74)$

1.00

$0.85(0.48-1.52)$
1.00

1.00

$0.77(0.45-1.32)$

$0.74(0.28-1.92)$

1.00

1.00

$1.08(0.35-3.34)$

$1.50(0.39-5.85)$

$0.70(0.33-1.50)$

$0.67(0.28-1.64)$

$0.57(0.33-1.00) \quad 0.57(0.30-1.10)$

1.00

1.00

$0.71(0.40-1.23)$

$0.80(0.44-1.48)$

1.00

1.00

$1.10(0.62-1.96)$

$1.21(0.65-2.27)$

$1.36(0.74-2.51)$

$1.72(0.91-3.28)$

1.00

1.00

$0.86(0.50-1.50)$

$0.86(0.46-1.59)$

$0.68(0.43-1.09)$

$0.69(0.39-1.22)$

1.00

1.00 $0.66(0.31-1.42)$ 
Table 3 Associations (odds ratios) between social, demographic, perinatal factors and suicide risk or self-harm. (Continued)

\begin{tabular}{|c|c|c|c|c|c|c|}
\hline $6+$ years or singleton & $0.92(0.55-1.55)$ & $1.25(0.54-2.93)$ & $0.95(0.58-1.57)$ & $1.15(0.54-2.46)$ & $0.96(0.59-1.56)$ & $0.67(0.26-1.70)$ \\
\hline \multicolumn{7}{|l|}{ Maternal age (years) } \\
\hline $15-19$ & $1.84(0.92-3.67)$ & $1.25(0.59-2.66)$ & $1.68(0.90-3.11)$ & $1.27(0.65-2.49)$ & $0.92(0.31-2.74)$ & $0.86(0.28-2.65)$ \\
\hline $20-24$ & $1.25(0.79-2.00)$ & $1.00(0.58-1.71)$ & $1.20(0.81-1.78)$ & $1.05(0.66-1.66)$ & $1.11(0.65-1.89)$ & $1.04(0.58-1.88)$ \\
\hline $25-29$ & 1.00 & 1.00 & 1.00 & 1.00 & 1.00 & 1.00 \\
\hline $30-34$ & $0.82(0.45-1.51)$ & $0.86(0.45-1.66)$ & $1.04(0.66-1.64)$ & $1.04(0.63-1.73)$ & $1.19(0.73-1.96)$ & $1.23(0.74-2.07)$ \\
\hline $35-46$ & $1.09(0.47-2.53)$ & $1.29(0.51-3.24)$ & $1.25(0.62-2.53)$ & $1.44(0.68-3.08)$ & $1.03(0.45-2.37)$ & $1.02(0.38-2.74)$ \\
\hline \multicolumn{7}{|l|}{ Breastfeeding } \\
\hline None & 1.00 & 1.00 & 1.00 & 1.00 & 1.00 & 1.00 \\
\hline Less than 6 months & $0.78(0.43-1.43)$ & $0.94(0.49-1.79)$ & $0.67(0.40-1.13)$ & $0.75(0.43-1.33)$ & $0.90(0.51-1.60)$ & $0.96(0.48-1.88)$ \\
\hline 6 months or more & $0.63(0.34-1.16)$ & $0.87(0.42-1.80)$ & $0.75(0.47-1.18)$ & $0.87(0.52-1.45)$ & $0.89(0.48-1.68)$ & $0.90(0.47-1.74)$ \\
\hline
\end{tabular}

Significant odds ratios ( $p=0.05$ or lower) are shown in bold. Final sample with multiple imputation $=2157$.

$\Theta_{11}=$ participant provided information at age $11 ; @ 15=$ information provided at age $15 ;{ }^{@ 19}=$ information provided at age 19 ; ${ }^{@ 11 p}=$ parental figure provided information when participants age 11.

association may be stronger among specific types of psychiatric disorder; a future study will look at the associations between birth complication more specific psychiatric disorders and symptoms, such as ADHD.

\section{Lack of associations with perinatal circumstances and mental heath outcomes}

Objectively, we found few associations between perinatal circumstances and mental health outcomes and can only speculate as to the reasons for the predominantly null findings. One explanation for the lack of statistically significant associations with perinatal variables may be the smaller power in this study compared with population based studies. This may be especially relevant for groups such as those with birth weights below $2500 \mathrm{~g}$, where the case numbers were small. The data do not indicate any consistent trend towards the risks associated with perinatal variables being attenuated on multivariable analysis, suggesting that controlling for adolescent psychosocial factors does not account for this lack of association.

An alternative explanation may be provided by the equalisation in youth hypothesis which suggests that during adolescence young people with different socioeconomic backgrounds demonstrate relative parity of health [38], it being only later in life that health inequalities (re)emerge. Whereas other studies on perinatal influences followed up subjects to their fourth decade, this study has, (so far) not collected any outcome data beyond early adulthood.

A notable exception to the apparent lack of perinatal influence is the case of young maternal age, for which the data does suggest a trend towards an attenuation of the associated risks following adjustment. This suggests that previously reported associations may have been mediated, in part at least, by more proximal adolescent psychosocial factors such as family structure and parental relationships.

\section{Associations between psychosocial circumstances and mental health outcomes}

Those from a single parent family were at greater risk than those living with two biological parents, although such differences were not statistically significant and there was a trend for these risks to attenuate after adjustment for other factors. Those from a "reconstituted family" were at significantly greater risk, although this also attenuated somewhat following adjustment. These observations appear consistent with a recent New Zealand study, which concluded that the associations between exposure to a single parent family and adversity can be attributed to the social environment [20]. In contrast to family structure, perceived deficiency in parental care was associated with increased risk which was minimally attenuated following adjustment. These observations seem consistent with other studies which suggest it is the quality and stability of the parent child relationship or family function which is the risk mediator $[19,20,39]$, whereas family structure is a more distal risk factor.

The relationships between same sex partner and suicidality, although statistically significant only in the case of NSSI at age 19, are attenuated following adjustment. This is consistent with the more likely interpretation of sexual orientation as a risk indicator rather than mediator i.e. the increased psychiatric risk is mediated by associated sociological, psychological and cultural factors, such as victimisation or identity issues.

The association between mental health outcome and same sex partner is consistent with previous studies on the relationship between mental health and homosexuality. While such an association between sexual orientation and risk of suicide or NSSI is well-established $[40,41]$, another robust association is that between birth order (specifically the number of older brothers) and male homosexual orientation [42]. The leading 
Table 4 Associations (odds ratios) between social, demographic, perinatal factors and use of psychological services.

\begin{tabular}{|c|c|c|c|c|c|c|}
\hline \multirow[b]{2}{*}{ Covariates } & \multicolumn{2}{|c|}{ Psych service use before age- 11} & \multicolumn{2}{|c|}{ Psych service use after 11} & \multicolumn{2}{|c|}{ ANY Psych service use } \\
\hline & Unadjust & Adjusted & Unadjust & Adjusted & Unadjust & Adjusted \\
\hline \multicolumn{7}{|l|}{ Psychosocial } \\
\hline \multicolumn{7}{|l|}{ Sex @11 } \\
\hline Male & 1.00 & 1.00 & 1.00 & 1.00 & 1.00 & 1.00 \\
\hline Female & $0.38(0.23-0.62)$ & $0.35(0.21-0.59)$ & $2.07(1.09-3.91)$ & $2.11(1.08-4.11)$ & $0.91(0.53-1.56)$ & $0.93(0.56-1.56)$ \\
\hline \multicolumn{7}{|l|}{ Social class @11p } \\
\hline Non-manual & 1.00 & 1.00 & 1.00 & 1.00 & 1.00 & 1.00 \\
\hline Manual & $1.22(0.69-2.16)$ & $1.34(0.70-2.54)$ & $1.44(0.59-3.51)$ & $1.23(0.60-2.54)$ & $1.43(0.88-2.32)$ & $1.15(0.75-1.76)$ \\
\hline \multicolumn{7}{|l|}{ Family structure @15 } \\
\hline 2-parent & 1.00 & 1.00 & 1.00 & 1.00 & 1.00 & 1.00 \\
\hline 1-parent, & $1.05(0.47-2.33)$ & $0.96(0.41-2.25)$ & $2.08(0.99-4.39)$ & $2.05(0.99-4.25)$ & $1.47(0.78-2.78)$ & $1.48(0.80-2.73)$ \\
\hline reconstituted/Other & $2.00(1.00-4.00)$ & $1.48(0.68-3.23)$ & $2.24(0.87-5.77)$ & $2.93(1.35-6.36)$ & $2.15(1.22-3.79)$ & $2.61(1.58-4.30)$ \\
\hline \multicolumn{7}{|l|}{ Same sex part ${ }^{@ 19}$} \\
\hline No & 1.00 & 1.00 & 1.00 & 1.00 & 1.00 & 1.00 \\
\hline Yes & NIL & NIL & $7.10(3.29-15.33)$ & 7.34 (3.44-15.64) & $3.49(1.72-7.11)$ & $3.42(1.51-7.77)$ \\
\hline \multicolumn{7}{|l|}{ Gender conform @15 } \\
\hline Gender typical & 1.00 & 1.00 & 1.00 & 1.00 & 1.00 & 1.00 \\
\hline Gender conformist & $0.50(0.15-1.62)$ & $0.42(0.13-1.41)$ & $0.85(0.20-3.66)$ & $0.86(0.23-3.19)$ & $0.80(0.28-2.30)$ & $0.92(0.35-2.40)$ \\
\hline Gender nonconformist & $1.00(0.43-2.32)$ & $0.90(0.38-2.10)$ & $0.67(0.27-1.68)$ & $1.28(0.51-3.19)$ & $1.04(0.55-1.96)$ & $1.56(0.83-2.94)$ \\
\hline
\end{tabular}
Religion@11p

\begin{tabular}{ccccccc} 
Protestant & 1.00 & 1.00 & 1.00 & 1.00 & 1.00 & 1.00 \\
Roman Catholic & $0.65(0.38-1.13)$ & $0.66(0.37-1.17)$ & $1.17(0.54-2.54)$ & $1.08(0.54-2.16)$ & $0.84(0.45-1.60)$ & $0.84(0.48-1.47)$ \\
Other (Baptist, etc) & $0.50(0.18-1.42)$ & $0.52(0.18-1.51)$ & $1.48(0.42-5.28)$ & $1.26(0.43-3.70)$ & $1.23(0.60-2.51)$ & $1.11(0.55-2.24)$ \\
None/Atheist/Agnostic & $1.40(0.80-2.46)$ & $1.16(0.64-2.11)$ & $1.19(0.43-3.24)$ & $1.38(0.54-3.52)$ & $0.83(0.29-2.40)$ & $1.05(0.39-2.83)$ \\
\hline Area deprivation ${ }^{@ 11}$ & $0.97(0.85-1.11)$ & $0.91(0.78-1.06)$ & $0.91(0.75-1.10)$ & $0.97(0.83-1.13)$ & $0.89(0.75-1.06)$ & $0.95(0.83-1.10)$ \\
Lack of par Care ${ }^{\circledR 15}$ & $1.14(0.98-1.32)$ & $1.13(0.97-1.32)$ & $\mathbf{1 . 2 6 ( 1 . 0 1 - 1 . 5 6 )}$ & $\mathbf{1 . 2 9 ( 1 . 0 7 - 1 . 5 5 )}$ & $1.12(0.881 .42)$ & $1.15(0.92-1.45)$ \\
Parental control @15 & $1.19(0.95-1.50)$ & $1.14(0.90-1.44)$ & $0.96(0.68-1.35)$ & $0.98(0.73-1.32)$ & $1.24(0.94-1.63)$ & $1.24(0.97-1.57)$
\end{tabular}

Perinatal all @11p

Maternal parity

0

1 or more

Family size

2

3 or more

Birth weight (g)

$<2500$
$2500-3249$
$3250-3749$
$3750+$

N birth complications

0

1

2 or more

Birth spacing (prior)

Under 2 years

2-5 years

$6+$ years or singleton

Birth spacing (later)

Under 2 years

2-5 years

$6+$ years or singleton
1.00

$0.58(0.37-0.91)$

1.00

$0.33(0.20-0.54) \quad 0.45(0.20-1.02)$

$1.43(0.57-3.55)$

$1.38(0.81-2.37)$

1.00

$1.49(0.81-2.75)$

$1.40(0.53-3.71)$

$1.42(0.81-2.49)$

1.00

$1.43(0.75-2.72)$

1.00

1.00

$0.81(0.41-1.61)$

$0.73(0.36-1.50)$

$2.26(1.34-3.82)$

$2.29(1.30-4.03)$

$\begin{array}{cc}1.00 & 1.00 \\ 2.02(0.84-4.84) & 2.03(0.83-4.99) \\ 2.14(0.96-4.74) & 1.76(0.72-4.34)\end{array}$

1.00

$0.71(0.33-1.56)$

1.00

$1.44(0.79-2.62)$
$0.71(0.32-1.57)$

$1.22(0.49-3.04)$
1.00

$0.91(0.19-4.23)$

1.00

$0.46(0.13-1.59)$

$0.74(0.21-2.61)$

$0.66(0.29-1.49)$

1.00

$0.70(0.32-1.53)$

1.00

$1.58(0.75-3.31)$

$2.63(0.87-7.94)$

1.00

$0.76(0.28-2.11)$

$0.39(0.17-0.88)$

1.00

$0.37(0.10-1.32)$

0.41 (0.08-1.99)
1.00

$0.80(0.44-1.49)$

1.00

$0.66(0.25-1.71)$

$0.90(0.29-2.84)$

0.67 (0.33-1.37)

1.00

$0.58(0.29-1.16)$

1.00

$1.24(0.66-2.34)$

$1.67(0.59-4.72)$

1.00

$0.68(0.28-1.64)$

$0.56(0.26-1.21)$

1.00

$0.38(0.11-1.23)$

0.70 (0.33-1.49)
1.00

$1.21(0.43-3.37)$

1.00

$0.69(0.40-1.21)$

1.00

$0.38(0.13-1.10)$

$0.43(0.21-0.88)$

$1.14(0.36-3.63)$

$0.92(0.56-1.50)$

1.00

$0.93(0.51-1.70)$

$1.36(0.47-3.94)$

$0.98(0.59-1.63)$

1.00

$0.88(0.51-1.54)$

1.00

$1.92(1.02-3.62)$

$3.77(1.83-7.76)$

1.00

$1.68(0.92-3.10)$

$2.92(1.45-5.88)$

1.00

1.00

$0.94(0.48-1.82)$

$0.90(0.46-1.76)$

$0.91(0.42-1.94)$

$0.61(0.21-1.73)$

1.00

$0.57(0.27-1.20)$

$0.56(0.27-1.16)$

$0.88(0.32-2.42)$

$1.05(0.62-1.76)$ 
Table 4 Associations (odds ratios) between social, demographic, perinatal factors and use of psychological services. (Continued)

\begin{tabular}{|c|c|c|c|c|c|c|}
\hline \multicolumn{7}{|l|}{ Maternal age (years) } \\
\hline $15-19$ & $2.22(1.00-4.94)$ & $1.60(0.63-4.03)$ & $0.74(0.14-4.07)$ & $0.77(0.12-4.78)$ & $1.03(0.37-2.91)$ & $1.29(0.57-2.93)$ \\
\hline $20-24$ & $1.55(0.87-2.75)$ & $1.71(0.92-3.18)$ & $1.26(0.52-3.03)$ & $1.26(0.55-2.85)$ & $1.74(1.00-3.03)$ & $1.59(0.93-2.72)$ \\
\hline $25-29$ & 1.00 & 1.00 & 1.00 & 1.00 & 1.00 & 1.00 \\
\hline $30-34$ & $1.64(0.88-3.04)$ & $1.48(0.77-2.84)$ & $1.06(0.41-2.74)$ & $0.95(0.41-2.25)$ & $1.61(0.77-3.41)$ & $1.53(0.77-3.05)$ \\
\hline $35-46$ & $0.52(0.12-2.26)$ & $0.48(0.11-2.15)$ & $0.74(0.06-8.86)$ & $0.77(0.10-6.08)$ & $0.91(0.24-3.48)$ & $0.97(0.28-3.37)$ \\
\hline \multicolumn{7}{|l|}{ Breastfeeding } \\
\hline None & 1.00 & 1.00 & 1.00 & 1.00 & 1.00 & 1.00 \\
\hline Less than 6 months & $0.92(0.52-1.60)$ & $0.92(0.50-1.69)$ & $1.05(0.46-2.37)$ & $0.94(0.50-1.77)$ & $0.88(0.42-1.84)$ & $0.86(0.43-1.72)$ \\
\hline 6 months or more & $0.83(0.43-1.59)$ & $1.05(0.50-2.21)$ & $1.33(0.61-2.89)$ & $1.09(0.56-2.15)$ & $1.21(0.59-2.48)$ & $1.01(0.55-1.86)$ \\
\hline
\end{tabular}

Significant odds ratios ( $p=0.05$ or lower) are shown in bold. Final sample with multiple imputation $=2157$.

$@ 11=$ participant provided information at age $11 ;{ }^{@ 15}=$ information provided at age $15 ;{ }^{@ 19}=$ information provided at age 19; ${ }^{@ 11 p}=$ parental figure provided information when participants age 11.

explanation for this association is the $\mathrm{H}-\mathrm{y}$ antigen theory [43], which posits that a progressively stronger immunological response to each male foetus within the intrauterine environment plays a role in determining sexual orientation. Previously reported associations between birth order and suicidality $[13,14]$ may therefore be mediated by sexual orientation, with birth order being merely a risk indicator. However, in the absence of any apparent relationship in this study, between maternal parity and outcome, even before adjustment, it is not possible to comment on the extent to which sexual orientation influences our findings, although the 'elder brother effect' may have confounded previous observations on maternal parity.

\section{Limitations of study}

Much of the data is self reported, and so it may be subject to recall bias. The data available on parental care and control, and on family structure, was cross sectional information, which was collected at age 15 . Length of exposure to such factors during the course of childhood was not available, and so not available for analysis. Although the study provided a rich dataset, the power of the study to identify associations between perinatal circumstances and mental health outcomes was limited, and this should be borne in mind in the interpretation of the lack of association in the present work.

Our study focused on a limited set of narrowly operationalized outcomes, potentially losing insight about the link between perinatal circumstances and severity of psychiatric symptoms and specific psychiatric disorders; further work to explore these links is required. Data involving the use of psychiatric services, especially before the age of 11, ought to be interpreted with some caution as these variables may be greatly influenced by parental attitude and behaviour and lack sensitivity when measuring the degree of psychiatric symptomatology. It therefore may not be a valid measure of prevalence of mental disorders. For example, parents with small families may be more likely to seek psychiatric help for their young children than are those of large families. Observations of relationships between service use and variables such as family size, pregnancy spacing and maternal parity, should be interpreted with this in mind. However, data on suicidal ideation, suicide attempt and NSSI, although self reported, are not likely to be influenced by parental behaviour as it is would only be indirectly influenced by parental help-seeking.

The data available on parental care and control, and on family structure, was cross sectional information, which was collected at age 15 . Length of exposure to such factors during the course of childhood was not available, and so not available for analysis. Although the study provided a rich dataset, the power of the study to identify associations between perinatal circumstances and mental health outcomes was limited, and this should be borne in mind in the interpretation of the lack of association in the present work.

\section{Conclusion}

A number of adolescent psychosocial variables may be risk factors for suicidality which could be the focus of preventative measures, the most notable being the quality of parent child relationships. Further research is necessary to clarify the role of variables such as sexual orientation and birth trauma in the development of suicidal behaviour. Although we echo Tharpar \& Rutter's [21] caution in ascribing perinatal factors a causal role in the development of psychiatric problems, the strong relationship we find between obstetric complications in this population sample and psychiatric service use, which adjusts for a range of likely confounds, re-opens the debate in this area.

\section{Acknowledgements}

The authors thank all those who made the 11-16/16+ study possible, and all the schools, survey assistants and young people who took part. We would 
like to thank Chris Lucas for his invaluable contribution regarding the VoiceDISC interview software.

\section{Author details}

${ }^{1}$ MRC Social and Public Health Sciences Unit, University of Glasgow, 4 Lilybank Gardens, Glasgow G12 8RZ, Scotland, UK. ${ }^{2}$ West Cork Mental Health Services, HSE. Bantry, Co. Cork, Ireland; Centre for Rural Realth Research and Policy, Inverness, Scotland, UK. ${ }^{3} \mathrm{NHS}$ Highland, Inverness, Scotland, UK, Centre for Rural Health Research and Policy, Inverness, Scotland, UK.

\section{Authors' contributions}

All authors contributed to the writing of the manuscript and jointly conceived the theoretical approach taken in the paper. RY participated in design and data collection of the final phase of the 11-16/16+ study, and performed the statistical analysis. Conceptually VR and CS primarily focused on perinatal and RY the social influences. All authors read and approved the final manuscript.

\section{Competing interests}

RY is supported financially by the Medical Research Council of Great Britain at the Social and Public Health Sciences Unit as part of the Youth and Health Programme (WBS U.1300.00.007). The authors declare no other competing interests.

Received: 28 January 2011 Accepted: 18 November 2011 Published: 18 November 2011

\section{References}

1. Rutter M: Environmentally mediated risks for psychopathology: research strategies and findings. J Am Acad Child Psychiatry 2005, 44(1):3-18.

2. Rutter M: How the environment affects mental health. Br J Psychiatry 2005, 186(1):4-6.

3. Bowlby J: The making and breaking of affectional bonds. I. Aetiology and psychopathology in the light of attachment theory. An expanded version of the Fiftieth Maudsley Lecture, delivered before the Royal College of Psychiatrists, 19 November 1976. Br J Psychiatry 1977, 130:201-210.

4. Richards M, Hardy R, Wadsworth M: The effects of divorce and separation on mental health in a national UK birth cohort. Psychol Med 1997, 27(5):1121-1128.

5. Browne A, Finkelhor D: Impact of Child Sexual Abuse - a Review of the Research. Psychol Bull 1986, 99(1):66-77.

6. Scott KM, Smith DR, Ellis PM: Prospectively Ascertained Child Maltreatment and Its Association With DSM-IV Mental Disorders in Young Adults. Arch Gen Psychiatry 2010, 67(7):712-719.

7. Mullen PE, Martin JL, Anderson JC, Romans SE, Herbison GP: Childhood sexual abuse and mental health in adult life. Br J Psychiatry 1993, 163:721-732.

8. Enns MW, Cox BJ, Afifi TO, De Graaf R, Ten Have M, Sareen J: Childhood adversities and risk for suicidal ideation and attempts: a longitudinal population-based study. Psychol Med 2006, 36(12):1769-1778.

9. Lipschitz DS, Winegar RK, Nicolaou AL, Hartnick E, Wolfson M, Southwick SM: Perceived abuse and neglect as risk factors for suicidal behavior in adolescent inpatients. J Nerv Ment Dis 1999, 187(1):32-39.

10. Brown AS, van Os J, Driessens C, Hoek HW, Susser ES: Further Evidence of Relation Between Prenatal Famine and Major Affective Disorder. Am J Psychiatry 2000, 157(2):190-195.

11. O'Keane V, Scott J: From 'obstetric complications' to a maternal-foetal origin hypothesis of mood disorder. Br J Psychiatry 2005, 186:367-368.

12. Thompson C, Syddall H, Rodin I, Osmond C, Barker DJ: Birth weight and the risk of depressive disorder in late life. Br J Psychiatry 2001, 179:450-455.

13. Gravseth HM, Mehlum L, Bjerkedal T, Kristensen P: Suicide in young Norwegians in a life course perspective: population-based cohort study. J Epidemiol Community Health 2010, 64(5):407-412.

14. Riordan DV, Selvaraj S, Stark C, Gilbert JS: Perinatal circumstances and risk of offspring suicide. Birth cohort study. Br J Psychiatry 2006, 189:502-507.

15. Mittendorfer-Rutz E, Rasmussen F, Wasserman D: Restricted fetal growth and adverse maternal psychosocial and socioeconomic conditions as risk factors for suicidal behaviour of offspring: a cohort study. Lancet 2004, 364(9440):1135-1140.
16. Barker DJ, Osmond C, Rodin I, Fall CH, Winter PD: Low weight gain in infancy and suicide in adult life. BMJ 1995, 311(7014):1203.

17. Tsankova N, Renthal W, Kumar A, Nestler EJ: Epigenetic regulation in psychiatric disorders. Nat Rev Neurosci 2007, 8(5):355-367.

18. Chase-Lansdale PL, Cherlin AJ, Kiernan KE: The long-term effects of parental divorce on the mental health of young adults: a developmental perspective. Child Dev 1995, 66(6):1614-1634.

19. O'Connor TG, Dunn J, Jenkins JM, Pickering K, Rasbash J: Family settings and children's adjustment: differential adjustment within and across families. Br J Psychiatry 2001, 179:110-115.

20. Fergusson DM, Boden JM, Horwood LJ: Exposure to single parenthood in childhood and later mental health, educational, economic, and criminal behavior outcomes. Arch Gen Psychiatry 2007, 64(9):1089-1095.

21. Thapar A, Rutter M: Do prenatal risk factors cause psychiatric disorder? Be wary of causal claims. Br J Psychiatry 2009, 195(2):100-101.

22. West $P$, Sweeting $H$ : Background, Rationale and Design of the West of Scotland 11 to 16 study. Working Paper No 53 Glasgow: MRC Social \& Public Health Sciences Unit; 1996

23. Ecob R, West $P$, Sweeting $H$ : The West of Scotland 11 to 16 Study: schools, sample design and implementation issues. Working Paper No 61 Glasgow: MRC Medical Sociology Unit; 1996.

24. West P, Sweeting H, Der G, Barton J, Lucas C: Voice-DISC identified DSM-IV disorders among 15-year-olds in the west of Scotland. J Am Acad Child Adolesc Psychiatry 2003, 42(8):941-949

25. Sweeting $H$, Der $G$, West $P$ : Bias, attrition and weighting in respect of the West of Scotland 11 to 16 Study's baseline, S2 and S4 surveys. Working Paper No 9 Glasgow: MRC Social \& Public Health Sciences Unit; 2001.

26. Section on Breastfeeding: Breastfeeding and the Use of Human Milk Pediatrics 2005, 115(2):496-506.

27. McLoone P: Carstairs scores for Scottish postcode sectors from the 2001 Census. Glasgow: MRC Social and Public Health Sciences Unit; 2004

28. ONS: In Standard Occupational Classification. Volume 2. London: The Stationery Office; 2000

29. Klimidis S, Minas $\mathrm{H}$, Ata AW: The PBI-BC: A brief current form of the Parental Bonding Instrument for adolescent research. Compr Psychiatry 1992, 33(6):374-377.

30. Young $\mathrm{R}$, Sweeting $\mathrm{H}$ : Adolescent bullying, relationships, psychological well-being, and gender-atypical behavior: A gender diagnosticity approach. Sex Roles 2004, 50(7-8):525-537.

31. Lippa RA: Gender-related traits in gay men, lesbian women, and heterosexual men and women: the virtual identify of homosexualheterosexual diagnosticity and gender diagnosticity. J Pers 2000, 68(5):899-926.

32. Eaton WW, Mortensen PB, Thomsen PH, Frydenberg M: Obstetric complications and risk for severe psychopathology in childhood. $J$ Autism Dev Disord 2001, 31(3):279-285.

33. Rutter M: Syndromes Attributed to Minimal Brain-Dysfunction in Childhood. Am J Psychiat 1982, 139(1):21-33.

34. Gillberg C: Deficits in attention, motor control, and perception: a brief review. Arch Dis Child 2003, 88(10):904-910.

35. Cannon M, Jones PB, Murray RM: Obstetric complications and schizophrenia: historical and meta-analytic review. Am J Psychiatry 2002, 159(7):1080-1092.

36. Scott J, McNeill Y, Cavanagh J, Cannon M, Murray R: Exposure to obstetric complications and subsequent development of bipolar disorder: Systematic review. Br J Psychiatry 2006, 189:3-11.

37. Neugebauer R, Reuss ML: Association of maternal, antenatal and perinatal complications with suicide in adolescence and young adulthood. Acta Psychiatr Scand 1998, 97(6):412-418.

38. West $P$ : Health inequalities in the early years: Is there equalisation in youth? Soc Sci Med 1997, 44(6):833-858.

39. Weich S, Patterson J, Shaw R, Stewart-Brown S: Family relationships in childhood and common psychiatric disorders in later life: systematic review of prospective studies. Br J Psychiatry 2009, 194(5):392-398.

40. Skegg K, Nada-Raja S, Dickson N, Paul C, Williams S: Sexual orientation and self-harm in men and women. Am J Psychiatry 2003, 160(3):541-546.

41. Fergusson DM, Horwood $\mathrm{L}$, Beautrais $\mathrm{AL}$ : Is sexual orientation related to mental health problems and suicidality in young people? Arch Gen Psychiatry 1999, 56(10):876-880.

42. Blanchard R, Bogaert AF: Homosexuality in men and number of older brothers. Am J Psychiatry 1996, 153(1):27-31. 
43. Blanchard R: Fraternal birth order and the maternal immune hypothesis of male homosexuality. Horm Behav 2001, 40(2):105-114.

\section{Pre-publication history}

The pre-publication history for this paper can be accessed here:

http://www.biomedcentral.com/1471-2458/11/875/prepub

doi:10.1186/1471-2458-11-875

Cite this article as: Young et al:: Perinatal and psychosocial

circumstances associated with risk of attempted suicide, non-suicidal

self-injury and psychiatric service use. A longitudinal study of young

people. BMC Public Health 2011 11:875.

Submit your next manuscript to BioMed Central and take full advantage of:

- Convenient online submission

- Thorough peer review

- No space constraints or color figure charges

- Immediate publication on acceptance

- Inclusion in PubMed, CAS, Scopus and Google Scholar

- Research which is freely available for redistribution

Submit your manuscript at www.biomedcentral.com/submit 